\title{
Bilateral coagulation of inferior hypophyseal artery and pituitary transposition during endoscopic endonasal interdural posterior clinoidectomy: do they affect pituitary function?
}

\author{
${ }^{*}$ Huy Q. Truong, MD, ${ }^{1}$ Hamid Borghei-Razavi, MD, PhD, ${ }^{1}$ Edinson Najera, MD, ${ }^{1}$ \\ Ana Carolina Igami Nakassa, MD, ${ }^{1}$ Eric W. Wang, MD, ${ }^{2}$ Carl H. Snyderman, MD, MBA, ${ }^{2}$ \\ Paul A. Gardner, MD, ${ }^{1}$ and Juan C. Fernandez-Miranda, MD' \\ Departments of ${ }^{1}$ Neurological Surgery and ${ }^{2}$ Otolaryngology, University of Pittsburgh Medical Center, Pittsburgh, Pennsylvania
}

OBJECTIVE The endoscopic endonasal transcavernous approach with interdural pituitary transposition provides surgical access to the posterior clinoids and interpeduncular cistern. Prior to posterior clinoidectomy, selective coagulation and transection of the inferior hypophyseal artery $(\mathrm{IHA})$ is recommended to prevent uncontrolled tearing of the artery and its avulsion from the wall of the cavernous carotid artery. The authors' preliminary experience has shown that unilateral sacrifice of the IHA caused no permanent endocrine dysfunction. In this study, they investigated the pituitary function in the setting of bilateral sacrifice of IHAs and pituitary transposition.

METHODS All patients with normal preoperative pituitary function who underwent endoscopic endonasal bilateral posterior clinoidectomy with bilateral IHA sacrifice between March 2010 and December 2016 were included and retrospectively evaluated. All data regarding pituitary function were collected. The degree of pituitary gland manipulation was estimated based on tumor size on preoperative MRI. An angle between a line from the point where the gland meets the floor of the sella to the highest point of the tumor and the horizontal plane of the sellar floor, or access angle, was also measured. Posterior pituitary bright spots on pre- and postoperative T1-weighted MRI were also reported.

RESULTS Twenty patients had bilateral transcavernous posterior clinoidectomies with coagulation of both IHAs. There were 13 chordomas, 3 epidermoid cysts, 2 chondrosarcomas, 1 meningioma, and 1 hemangiopericytoma. The mean follow-up was 19 months (range 13-84 months). Two patients experienced transient diabetes insipidus (DI) requiring desmopressin, which resolved before hospital discharge. One patient (with chordoma) developed delayed permanent DI, and a second patient (with hemangiopericytoma) developed permanent DI and panhypopituitarism. The access angle was higher in the group with pituitary dysfunction ( $47.25^{\circ}$ compared to $33.81^{\circ} ; p=0.07$ ). Posterior pituitary bright spots were preserved in $75 \%$ of cases with normal postoperative endocrine function.

CONCLUSIONS The endoscopic endonasal transcavernous approach to the interpeduncular cistern with pituitary transposition and bilateral sacrifice of the IHAs does not cause pituitary dysfunction in a majority of patients. When endocrine deficit occurs, it appears to be more likely to have been caused by surgical manipulation than loss of blood supply. This finding confirms clinically the crucial concept of interarterial anastomosis of pituitary vasculature proposed by anatomists.

https://thejns.org/doi/abs/10.3171/2018.2.JNS173126

KEYWORDS inferior hypophyseal artery; posterior clinoidectomy; diabetes insipidus; hypopituitarism; endoscopic endonasal transcavernous sinus; pituitary surgery

$\mathrm{T}$ HE inferior hypophyseal artery (IHA) is an important branch of the cavernous segment of the internal carotid artery (ICA). It can originate directly from the posterior bend of the cavernous ICA or as a branch of the meningohypophyseal trunk, which includes dorsal meningeal and tentorial arteries. The IHA runs from lateral to medial within the cavernous sinus, crossing anteriorly to the posterior clinoid to reach the inferior sellar surface and supply the posterior lobe of the pituitary gland and its capsule. ${ }^{9,11}$

ABBREVIATIONS $\mathrm{DI}=$ diabetes insipidus; $I C A=$ internal carotid artery; $I H A=$ inferior hypophyseal artery; SHA = superior hypophyseal artery. SUBMITTED December 13, 2017. ACCEPTED February 23, 2018.

${ }^{*}$ H.Q.T. and H.B.R. contributed equally to this study. 

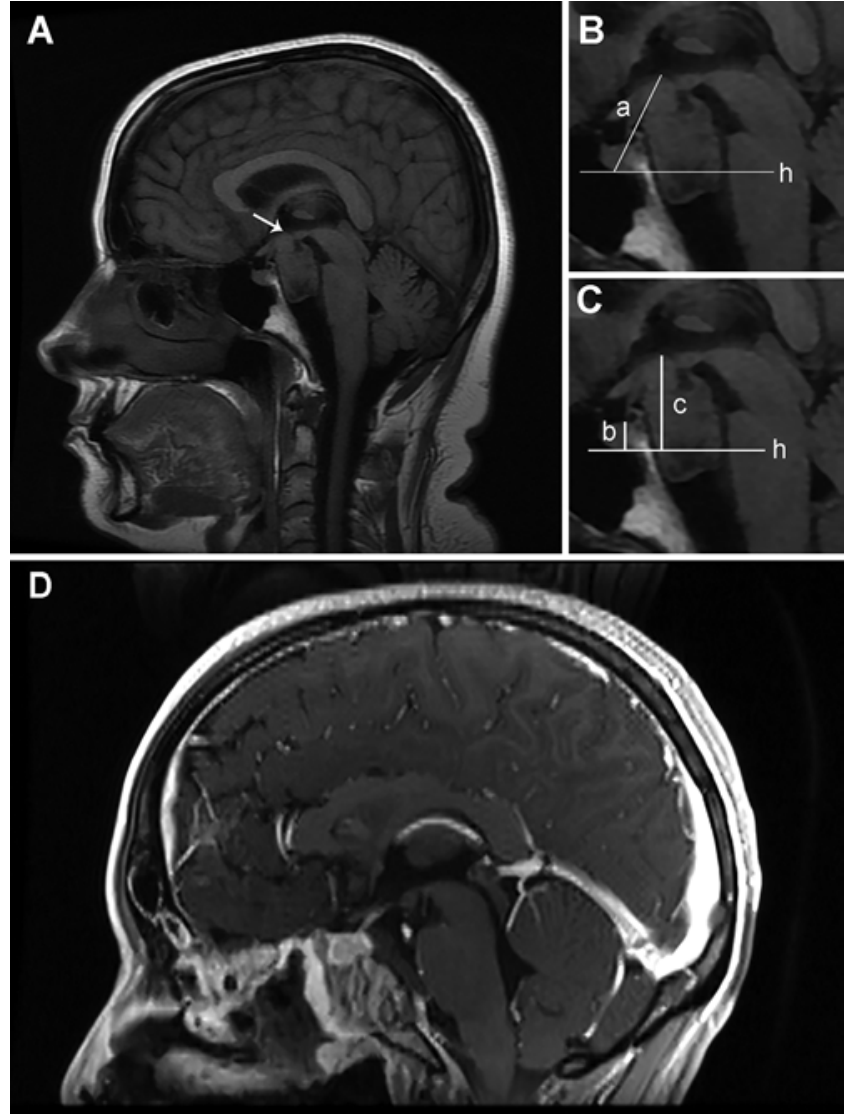

FIG. 1. Case 11. A: MR image of sagittal plane shows upper clival tumor, with high cranial extension of the lesion and compression on floor of the third ventricle (arrow). B: Measurement of access angle of the tumor, which is the angle between line a from the point where the gland meets the floor of the sella to the highest point of the tumor and the horizontal plane (line $h$ ) of the floor of the sella. C: Measurement of the height of pituitary gland (line $b$ ) compared to the cranial extension of tumor (line c) above the sellar floor (line $h$ ). D: Postoperative MR image of the case shows tumor removal and morphologically intact pituitary gland and thinned-out floor of the third ventricle.

Lying deep in the cavernous sinus and being well guarded laterally by the ICA, the IHA was rarely a surgical consideration until the endoscopic endonasal approach brought light via ventral access to the cavernous sinus. The endoscopic endonasal transcavernous approach with the interdural pituitary gland transposition technique provides excellent surgical access to the posterior clinoid and lateral interpeduncular cistern. ${ }^{3,4}$ Prior to performing a posterior clinoidectomy, elective coagulation and transection of the IHA is recommended to prevent uncontrolled tearing of the artery or its avulsion from the wall of the cavernous carotid artery, which could potentially be a serious complication with difficult-to-control intraoperative bleeding and postoperative development of ICA pseudoaneurysm. Our preliminary experience has shown that unilateral sacrifice of the IHA and pituitary transposition caused no permanent consequences to pituitary function. ${ }^{4}$ However, there are clinical situations in which bilateral interdural posterior clinoidectomies are required, such as extensive tumors invading the upper clivus and interpeduncular cistern bilaterally.
The purpose of this study was to evaluate the pituitary function in the event of bilateral sacrifice of the IHAs and pituitary transposition for bilateral posterior clinoidectomies during the endoscopic endonasal transcavernous approach. In this study, we challenge the concept of the IHAs as critical vessels for the vascular supply of the posterior lobe of the pituitary gland.

\section{Methods}

A retrospective review was conducted on the Cranial Base Center's medical records to include all patients who underwent endoscopic endonasal bilateral posterior clinoidectomy with bilateral IHA sacrifice between March 2010 and December 2016. A typical procedure is presented in Video 1.

VIDEO 1. Clip showing a typical posterior clinoidectomy procedure with bilateral coagulation of IHA. Copyright Juan C. Fernandez-

Miranda. Published with permission. Click here to view.

All data involving pituitary function were examined and recorded for analysis, including but not limited to postoperative fluid balance, urine sodium and density, serum sodium and osmolality, serum cortisol, pituitary (full hormone) panel, pathology, radiotherapy, and hormone replacement therapy. Hormone deficiency was deemed permanent if hormone replacement was still required at 6 months postoperatively. We excluded patients with preoperative hypopituitarism or with less than 6 months of follow-up.

Tumor dimensions were calculated on preoperative sagittal MRI. The cranial extension of the tumor above the sellar floor was recorded and compared to the height of the gland in percentage. An angle between a line from the point where the gland meets the floor of the sella to the highest point of the tumor and the horizontal plane of the sellar floor, or access angle, was also measured (Fig. 1).

The presence and location of the posterior pituitary bright spot on precontrast T1-weighted sequence MRI, which is thought to represent the vasopressin storage at the posterior lobe ${ }^{1}$ and is expected to be present in most patients, ${ }^{2}$ was reported pre- and postoperatively.

\section{Results}

During the study period, 23 patients underwent endonasal endoscopic transcavernous surgery with bilateral coagulation of the IHA as part of a bilateral posterior clinoidectomy. We excluded 2 cases due to previous pituitary dysfunction and 1 case with a follow-up time of less than 6 months. Among the 20 included cases, there were 13 chordomas, 3 epidermoid cysts, 2 chondrosarcomas, 1 meningioma, and 1 hemangiopericytoma. Six tumors were recurrent. The mean follow-up time was 19 months, ranging from 13 to 84 months. Preoperative measurement of cranial extension of tumors is presented in Table 1.

Four patients (20\%) developed diabetes insipidus (DI) in the postoperative period. Two of these patients (cases 8 and 17, Table 1) experienced transient DI (10\%) that required 1 to 3 doses of desmopressin daily and resolved before discharge from the hospital (5 and 8 days postoperatively). One patient (case 9) had a clival chordoma with intrasellar invasion, pushing the gland up and compress- 
TABLE 1. Pituitary function in 20 patients after bilateral posterior clinoidectomies with bilateral IHA coagulation and pituitary transposition

\begin{tabular}{|c|c|c|c|c|c|c|c|c|c|}
\hline \multirow{2}{*}{$\begin{array}{l}\text { Case } \\
\text { No. }\end{array}$} & \multirow{2}{*}{$\begin{array}{c}\text { Age (yrs) } \\
\text { Sex }\end{array}$} & \multirow[b]{2}{*}{ Pathology } & \multirow{2}{*}{$\begin{array}{c}\text { Cranial } \\
\text { Extension (\%) }\end{array}$} & \multirow{2}{*}{$\begin{array}{c}\text { Access } \\
\text { Angle }\end{array}$} & \multirow{2}{*}{$\begin{array}{l}\text { Ant Gland } \\
\text { Dysfunction }\end{array}$} & \multirow{2}{*}{$\begin{array}{c}\text { Transient DI } \\
\text { ( } \leq 6 \mathrm{mos})\end{array}$} & \multirow{2}{*}{$\begin{array}{l}\text { Permanent } \\
\text { DI (>6 mos) }\end{array}$} & \multicolumn{2}{|c|}{ Bright Spot } \\
\hline & & & & & & & & Preop & Postop \\
\hline 1 & $19, \mathrm{~F}$ & Chordoma & 112 & 38 & No & No & No & + & + \\
\hline 2 & $22, F$ & Chordoma & 124 & 22 & No & No & No & + & + \\
\hline 3 & $25, F$ & Chordoma & 108 & 17 & No & No & No & + & + \\
\hline 4 & $60, \mathrm{~F}$ & Epidermoid cyst & 184 & 28 & No & No & No & + & + \\
\hline 5 & $41, \mathrm{M}$ & Epidermoid cyst & 381 & 46 & No & No & No & + & + \\
\hline 6 & $20, F$ & Chordoma & 94 & 25 & No & No & No & + & + \\
\hline 7 & $24, \mathrm{M}$ & Chordoma & 171 & 35 & No & No & No & + & + \\
\hline 8 & $27, \mathrm{M}$ & Chordoma & 87 & 36 & No & Yes & No & + & + \\
\hline 9 & $25, \mathrm{~F}$ & Chordoma & 88 & 30 & No & Yes & Yes & + & - \\
\hline 10 & $28, M$ & Chordoma & 193 & 24 & No & No & No & + & + \\
\hline 11 & $52, \mathrm{~F}$ & Hemangiopericytoma & 329 & 65 & Yes & NA & Yes & + & - \\
\hline 12 & $61, F$ & Meningioma & 100 & 19 & No & No & No & + & - \\
\hline 13 & $33, F$ & Epidermoid cyst & 331 & 60 & No & No & No & + & + \\
\hline 14 & $40, M$ & Chordoma & 83 & 44 & No & No & No & + & + \\
\hline 15 & $67, \mathrm{M}$ & Chordoma & 298 & 41 & No & No & No & - & - \\
\hline 16 & $61, M$ & Chondrosarcoma & 215 & 32 & No & No & No & - & + \\
\hline 17 & $41, \mathrm{M}$ & Chordoma & 150 & 58 & No & Yes & No & + & - \\
\hline 18 & $73, \mathrm{~F}$ & Chondrosarcoma & 83 & 35 & No & No & No & + & + \\
\hline 19 & $33, \mathrm{M}$ & Chordoma & 115 & 31 & No & No & No & + & + \\
\hline 20 & $47, \mathrm{~F}$ & Chordoma & 145 & 44 & No & No & No & + & + \\
\hline
\end{tabular}

Ant $=$ anterior; $\mathrm{NA}=$ not applicable; + = present; - = absent.

ing it against the diaphragm and optic chiasm (Fig. 2). The posterior lobe was disrupted during tumor removal and the patient developed DI immediately postoperatively, but had normalized at discharge (postoperative day 3). She was later readmitted due to syndrome of inappropriate antidiuretic hormone (SIADH), which corrected after 3 days. Following a triphasic response, her last follow-up 4 months later confirmed the diagnosis of DI as permanent. Anterior lobe function, however, was intact.

The remaining patient had a large hemangiopericytoma (case 11, Table 1, Fig. 1) and developed both DI (which resolved at discharge-postoperative day 8) and adrenal insufficiency immediately after surgery. She developed DI again approximately 3 weeks postoperatively. Follow-up revealed panhypopituitarism that required long-term hormone replacement with desmopressin, hydrocortisone, and levothyroxine. The tumor in this case extended very cranially, with the highest angle of access and the third greatest height relative to the pituitary gland in this series (the 2 cases with the highest angles were epidermoid cysts); both
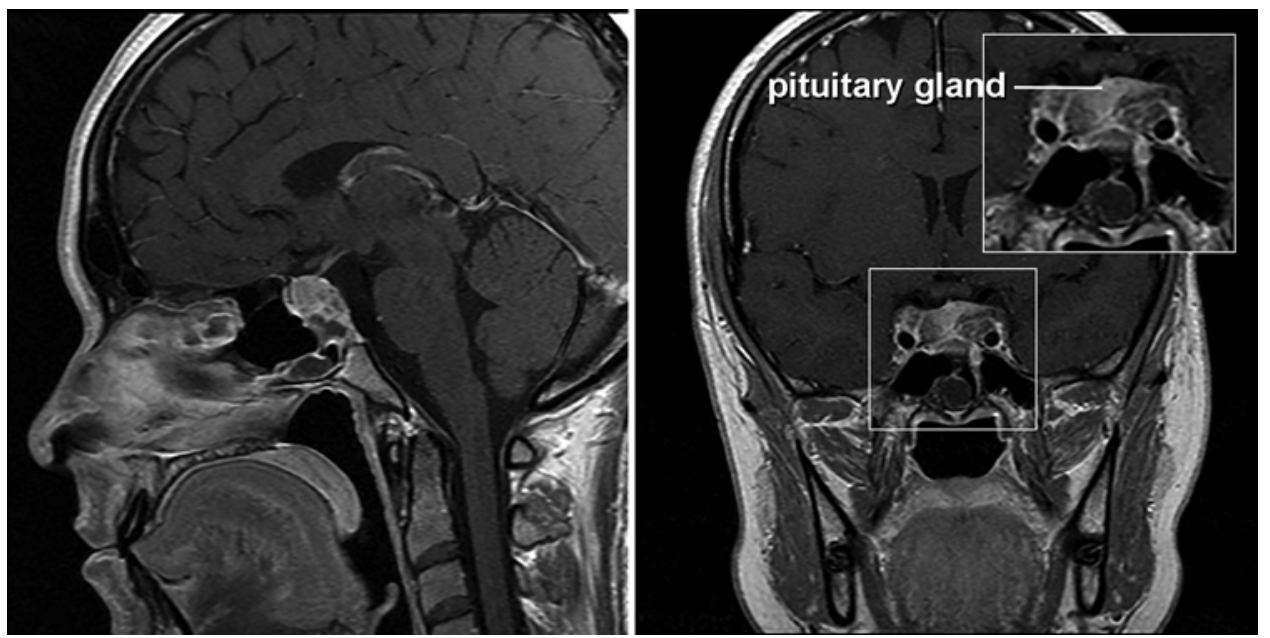

FIG. 2. Case 9. Left: Sagittal postcontrast T1-weighted MR image shows an upper clival tumor invading and filling up the sella. Right: Coronal postcontrast T1-weighted MR image showing the gland being compressed by the tumor against the diaphragma sellae. 

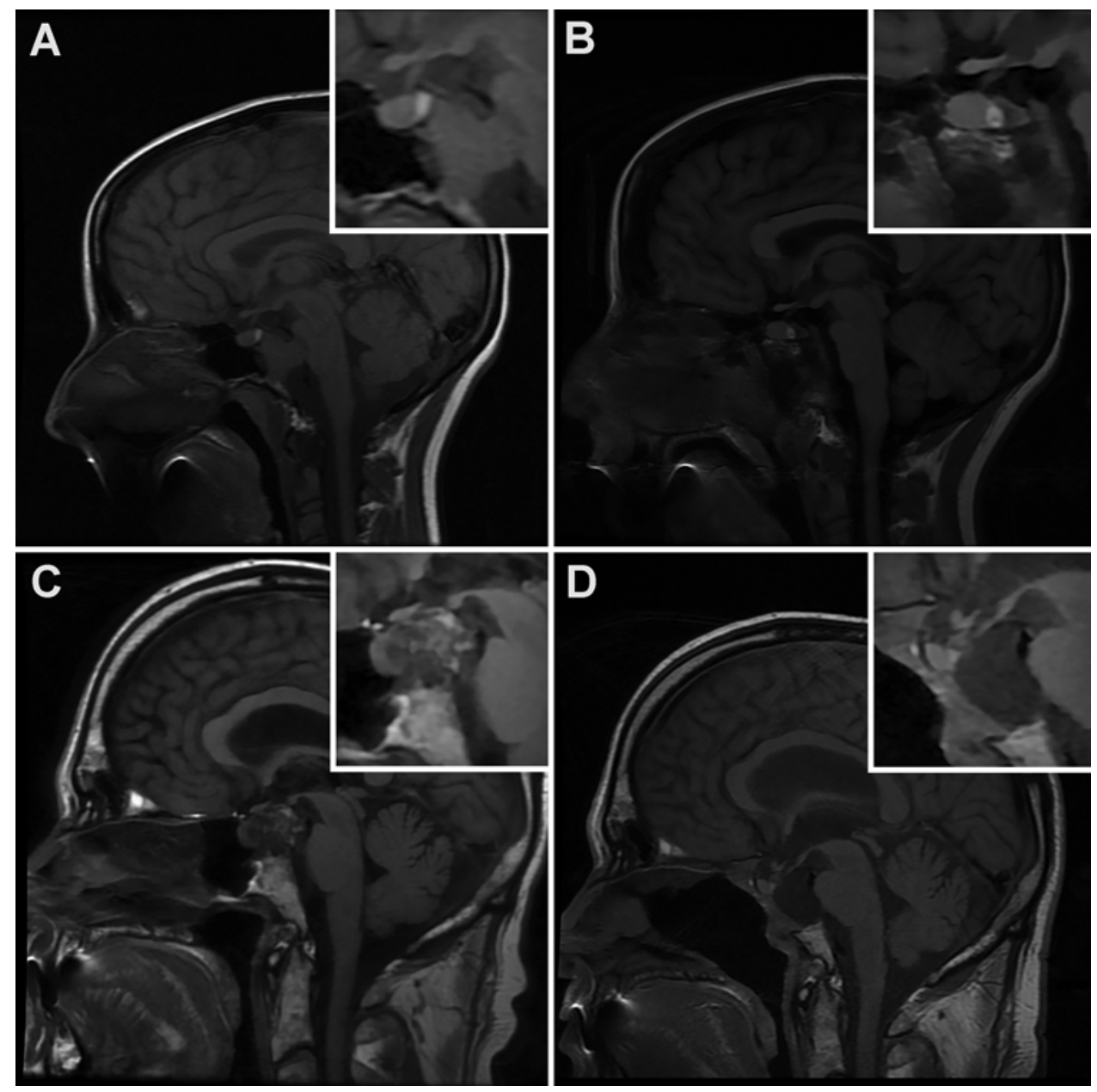

FIG. 3. Posterior pituitary bright spot (insets). A and B: Preoperative and postoperative sagittal T1-weighted MR images, respectively, of a clival chordoma case showing the preservation of the bright spot in the posterior lobe of the pituitary gland. C: Preoperative sagittal T1-weighted MR image of a case of chondrosarcoma that invaded the sellar floor, in which no bright spot can be identified. D: Postoperative imaging of the same plane and sequence reveals the bright spot.

conditions required extensive surgical manipulation of the pituitary gland and stalk, possibly leading to other devascularization, both arterial and venous.

Statistical analysis of tumor size and access angle measurements revealed that the relative size of cranial tumor extension to pituitary gland size is not different between the group with pituitary dysfunction (transient and permanent) and the rest of the group (163\% and $171 \%$, respectively). The access angle, which takes into consideration the anterior juxtaposition of tumor to pituitary gland, was higher in the group with pituitary dysfunction $\left(47.25^{\circ} \mathrm{com}-\right.$ pared to $33.81^{\circ}$ ); however, the difference did not reach statistical significance $(\mathrm{p}=0.071)$.

Preoperatively, the posterior pituitary bright spot was found in $18 / 20$ cases on its typical posterior sellar location (Fig. 3). There were 2 cases with no preoperative identification of the bright spot. Of the 18 cases with preoperative bright spot, 4 turned negative after tumor removal, and 2 of them developed permanent DI and 1 temporary DI. In the other 14 cases, the bright spots still resided in the posterior lobes. Of the 2 negative cases on preoperative imaging, neither developed DI, and in 1 of them (case 16) the bright spot was revealed after tumor removal (Fig. 3C and D). From these data, $75 \%$ of cases that lost the bright spot after surgery were associated with DI, whereas only 1 of 16 cases with identifiable bright spot on postoperative MRI developed transient DI.

No suprasellar approach with potential for injury to the superior hypophyseal artery (SHA) was performed in this series. There were no vascular complications (ICA injury) in this series. Postoperative radiotherapy was completed in 10 of 20 patients, including Gamma Knife and proton beam therapy, and none of them had developed new pituitary dysfunction at last follow-up.

\section{Discussion}

The pituitary stalk and gland receive blood supply from 2 arterial systems: the SHAs, which irrigate mainly the stalk and anterior lobe or adenohypophysis, and the IHAs, which supply mainly the posterior lobe or neurohypophysis. ${ }^{7,10,12}$ Bilateral compromise of the IHA alone could result in some degree of neurohypophysis dysfunction or DI, but adenohypophysis dysfunction is not theoretically 


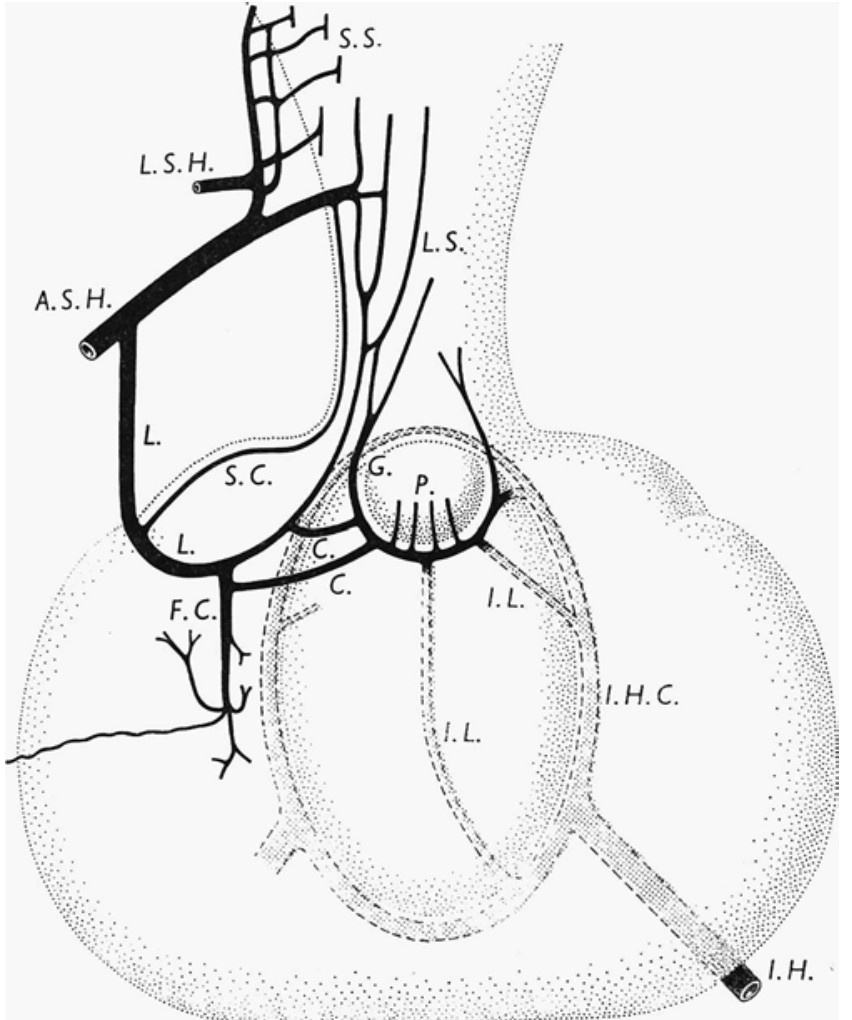

FIG. 4. Schematic illustration of interarterial connection between blood supplies of pituitary gland, by Stanfield JP in "The blood supply of the human pituitary gland." Journal of Anatomy. 1960; 94(Pt 2):257-273. (Courtesy of Wiley Publishing.) In this diagram, the IHAs from contralateral sides anastomose to form a vascular circle that sends out blood supply to the posterior lobe. The SHA from intracranial space provides blood supply to stalk and adenohypophysis through loral and long stalk arteries. Those arteries then connect to the vascular circle formed by IHAs. Original author's annotation: A.S.H. = anterior superior hypophyseal artery; $C$. = communicating arteries; F.C. = artery of fibrous core; G. = genual artery; I.H. = inferior hypophyseal artery; I.H.C. = inferior hypophyseal circle; I.L. = interlobar arteries; $L$. = loral artery; L.S. = long stalk arteries; $L . S . H .=$ lateral superior hypophyseal artery; $P$. = parallel vessels; S.C. = subcapsular artery; S.S. = short stalk arteries. (C) Anatomical Society, 1960, with permission of John Wiley \& Sons, Inc.

expected. The incidence of transient DI in this series of bilateral sacrifice of the IHAs is $20 \%$, the incidence of permanent DI is just $10 \%$, and the incidence of panhypopituitarism is $5 \%$. Based on these results, we can affirm that after bilateral sacrifice of the IHA the function of the neurohypophysis will be affected in a minority of cases. In fact, we hypothesize that the cause of DI in these patients is most likely related to surgical manipulation of the posterior lobe and stalk rather than ischemia secondary to vascular supply compromise. Intraoperative disruption of the posterior lobe was reported in the case with permanent DI (case 9), which may be the cause for her hormone deficiency. The case with panhypopituitarism and DI might be explained by a cause other than pure loss of blood supply from IHA occlusion. Gland injury due to manipulation during transposition was possibly the cause because the tumor extended very superiorly and anteriorly. Statistical analysis did not definitely confirm this hypothesis, but this could be secondary to the small sample size of this series.

Information on the clinical outcome of IHA occlusion is scant in the literature. Our search found only one report of transient DI after embolization of the meningohypophyseal trunk for treatment of a dural carotid-cavernous fistula. ${ }^{8}$ The occlusion of the IHA has been mentioned as a possible cause of Sheehan's syndrome in postpartum women. ${ }^{5}$ However, this statement is not supported by the current understanding of Sheehan's syndrome ${ }^{6}$ and the anatomy of the pituitary gland's blood supply.

The preservation of posterior pituitary bright spot in $79 \%$ of cases after bilateral coagulation of IHA is strong evidence that the neurohypophysis function was preserved and still resided in the posterior lobe of the pituitary gland after the posterior clinoidectomy.

The explanation for the exceptional tolerance of the neurohypophysis function to compromise of blood supply may lie in the anatomy of the hypophyseal arteries. McConnell proposed the existence of anastomosis between interlobar arteries from the IHA and the long stalk arteries from the SHA. ${ }^{7}$ This concept was later confirmed by Xuereb et al. ${ }^{12}$ and Stanfield.$^{10}$ In brief, the basic pattern described is that the IHAs, after reaching the lateral aspect of the pituitary gland at approximately the interlobar groove, give rise to 2 branches, namely ascending and descending, or anterior and posterior. The 2 branches anastomose with their counterparts to create an anastomotic arterial circle in the interlobar connective tissue between the anterior and posterior lobes. From this arterial circle, smaller branches arise to supply mainly the posterior lobe and some part of the anterior lobe near the interlobar septa. From the SHA, the loral artery, or artery of the trabecular, and the long stalk arteries run down along the stalk and anastomose with each other and with their counterparts to form a "transverse anastomosis," which then connects with the arterial circle from the IHA through several communicating arteries and interlobar arteries (Fig. 4). The anastomosis may vary from the basic pattern in terms of number and size.

The richly interarterial anastomoses provide an explanation for the low incidence of posterior pituitary dysfunction in our series. After the occlusion of both IHAs, the posterior gland may receive blood supply from the SHA through the loral arteries and/or long stalk arteries through their anastomoses. Consequently, caution should be taken to protect and preserve the SHAs if suprasellar dissection is needed after bilateral IHA coagulation. This is particularly relevant when dealing with large retroclival lesions that have extension into the suprasellar space.

Although it is possible to occasionally dissect and preserve the IHA during the posterior clinoidectomy procedure, our results support its early sacrifice because there are minimal clinical consequences, and it eliminates the risk of ICA avulsion from accidental pulling of the IHA when performing the posterior clinoidectomy.

\section{Conclusions}

The endoscopic endonasal transcavernous approach with bilateral posterior clinoidectomy, bilateral sacrifice of the IHAs, and pituitary transposition does not cause pituitary dysfunction in the vast majority of patients. This 
finding confirms clinically the current understanding of pituitary vasculature, highlighting the crucial role and characteristic features of the interarterial anastomosis of pituitary vasculature.

\section{References}

1. Colombo N, Berry I, Kucharczyk J, Kucharczyk W, de Groot J, Larson T, et al: Posterior pituitary gland: appearance on MR images in normal and pathologic states. Radiology 165:481-485, 1987

2. Côté M, Salzman KL, Sorour M, Couldwell WT: Normal dimensions of the posterior pituitary bright spot on magnetic resonance imaging. J Neurosurg 120:357-362, 2014

3. Essayed WI, Singh H, Lapadula G, Almodovar-Mercado GJ, Anand VK, Schwartz TH: Endoscopic endonasal approach to the ventral brainstem: anatomical feasibility and surgical limitations. J Neurosurg 127:1139-1146, 2017

4. Fernandez-Miranda JC, Gardner PA, Rastelli MM Jr, PerisCelda M, Koutourousiou M, Peace D, et al: Endoscopic endonasal transcavernous posterior clinoidectomy with interdural pituitary transposition. J Neurosurg 121:91-99, 2014

5. Greenberg MS: Handbook of Neurosurgery, ed 8. New York: Thieme, 2016, p 79

6. Keleştimur F: Sheehan's syndrome. Pituitary 6:181-188, 2003

7. McConnell EM: The arterial blood supply of the human hypophysis cerebri. Anat Rec 115:175-203, 1953

8. Phatouros CC, Higashida RT, Malek AM, Smith WS, Dowd CF, Halbach VV: Embolization of the meningohypophyseal trunk as a cause of diabetes insipidus. AJNR Am J Neuroradiol 20:1115-1118, 1999

9. Reisch R, Vutskits L, Patonay L, Fries G: The meningohypophyseal trunk and its blood supply to different intracranial structures. An anatomical study. Minim Invasive Neurosurg 39:78-81, 1996

10. Stanfield JP: The blood supply of the human pituitary gland. J Anat 94:257-273, 1960
11. Tran-Dinh H: Cavernous branches of the internal carotid artery: anatomy and nomenclature. Neurosurgery 20:205-210, 1987

12. Xuereb GP, Prichard MML, Daniel PM: The arterial supply and venous drainage of the human hypophysis cerebri. Q J Exp Physiol Cogn Med Sci 39:199-217, 1954

\section{Disclosures}

The authors report no conflict of interest concerning the materials or methods used in this study or the findings specified in this paper.

\section{Author Contributions}

Conception and design: Fernandez-Miranda, Borghei-Razavi. Acquisition of data: Truong, Borghei-Razavi, Najera, Igami Nakassa. Analysis and interpretation of data: Truong, BorgheiRazavi, Najera, Igami Nakassa. Drafting the article: Truong. Critically revising the article: Fernandez-Miranda, Truong, BorgheiRazavi, Najera, Wang, Snyderman, Gardner. Reviewed submitted version of manuscript: Fernandez-Miranda, Truong, BorgheiRazavi, Gardner. Approved the final version of the manuscript on behalf of all authors: Fernandez-Miranda. Statistical analysis: Truong. Study supervision: Fernandez-Miranda.

\section{Supplemental Information \\ Videos \\ Video 1. https://vimeo.com/263342815.}

\section{Correspondence}

Juan C. Fernandez-Miranda: Stanford University, Stanford, CA. drjfm@stanford.edu. 